\title{
BMJ
}

\section{Reliability of self reported smoking status by pregnant women for estimating smoking prevalence: a retrospective, cross sectional study}

\author{
Deborah Shipton, career development fellow, ${ }^{1}$ David M Tappin, director of paediatric epidemiology and \\ community health (PEACH) unit, ${ }^{2}$ Thenmalar Vadiveloo, PhD student, ${ }^{3}$ Jennifer A Crossley, consultant clinical \\ scientist, ${ }^{3}$ David A Aitken, consultant clinical scientist, ${ }^{3}$ Jim Chalmers, consultant in public health medicine ${ }^{4}$
}

${ }^{1}$ MRC Social and Public Health Sciences Unit, Glasgow G12 8RZ

${ }^{2}$ Paediatric Epidemiology and Community Health (PEACH) Unit, University of Glasgow, Yorkhill Hospital, Glasgow G3 8S) ${ }^{3}$ Institute of Medical Genetics, Yorkhill Hospital, Glasgow G3 8S)

${ }^{4}$ Information Services Division (ISD), NHS National Services Scotland, Edinburgh EH12 9EB

Correspondence to: D Tappin goda11@udcf.gla.ac.uk

Cite this as: $B M J$ 2009;339:b4347 doi:10.1136/bmj.b4347

\section{ABSTRACT}

Objective To determine what impact reliance on self reported smoking status during pregnancy has on both the accuracy of smoking prevalence figures and access to smoking cessation services for pregnant women in Scotland.

Design Retrospective, cross sectional study of cotinine measurements in stored blood samples.

Participants Random sample ( $n=3475)$ of the 21029 pregnant women in the West of Scotland who opted for second trimester prenatal screening over a one year period.

Main outcome measure Smoking status validated with cotinine measurement by maternal area deprivation category (Scottish Index of Multiple Deprivation). Results Reliance on self reported smoking status underestimated true smoking by $25 \%(1046 / 3475$ (30\%) from cotinine measurement $v 839 / 3475$ (24\%) from self reporting, $z$ score $8.27, \mathrm{P}<0.001)$. Projected figures suggest that in Scotland more than 2400 pregnant smokers go undetected each year. A greater proportion of smokers in the least deprived areas (deprivation categories 1+2) did not report their smoking (39\%) compared with women in the most deprived areas $(22 \%$ in deprivation categories $4+5$ ), but, because smoking was far more common in the most deprived areas (706 (40\%) in deprived areas compared with 142 (14\%) in affluent areas), projected figures for Scotland suggest that twice as many women in the most deprived areas are undetected $(n=1196)$ than in the least deprived areas $(n=642)$.

Conclusion Reliance on self reporting to identify pregnant smokers significantly underestimates the number of pregnant smokers in Scotland and results in a failure to detect over 2400 smokers each year who are therefore not offered smoking cessation services.

\section{INTRODUCTION}

Although the risks of smoking during pregnancy for both mother and child are well established, ${ }^{1}$ smoking during pregnancy is still common, with smoking rates varying from $24 \%$ in Scotland $^{2}$ to $17 \%$ in England. ${ }^{3}$
Smoking prevalence generally increases with deprivation, and this is certainly true of Scotland, where $41 \%$ of women in the most deprived areas report smoking compared with only $14 \%$ in the least deprived areas. ${ }^{4}$ Alarmingly, the gap in smoking prevalence between deprivation areas is larger in the pregnant population, where smoking is reduced to $8 \%$ in the least deprived areas but to only $38 \%$ in the most deprived area (data from the Scottish Morbidity Record (SMR02) for 2005, provided by NHS Information Services Division, www.isdscotland.org).

Self reported smoking is commonly used in antenatal clinics to determine the smoking status of pregnant women. However, this is an inaccurate method of identifying smokers, ${ }^{5}$ with studies suggesting up to a quarter of pregnant smokers are missed when self reporting is relied on.$^{6-8}$ The accuracy of self report varies by the setting in which the questions are asked. Routinely collected data, such as in antenatal care, is often less accurate than data collected in settings perceived as more neutral, such as in research interviews. ${ }^{9} \mathrm{With}$ mounting social and medical pressure on women to quit smoking during pregnancy, there is greater likelihood that pregnant women will not report their smoking. Such inaccuracy can affect the reliability of smoking prevalence figures and access to smoking cessation services.

National targets to improve the nation's health generally include targets to reduce smoking during pregnancy, ${ }^{1011}$ often with the explicit aim of reducing inequalities related to deprivation. The Scottish government uses self reported smoking at maternity booking to construct targets and to measure the success of services in reaching such targets. These measures need to be robust in order to assess if services are achieving their aim and if money is being well spent in trying to achieve these targets.

Smoking cessation interventions have been shown to be moderately effective in reducing continued smoking into late pregnancy, with a risk of smoking for the intervention group relative to the control group of $0.94 .^{12}$ Perhaps more importantly intervention is effective at reducing the proportion of babies 
of low birth weight (relative risk 0.81 (95\% CI 0.70 to $0.94))$ and of preterm births $(0.84(0.72$ to 0.98$))$, and at increasing birth weight (33 $\mathrm{g}$ (11 g to $55 \mathrm{~g}$ ) increase in weight). ${ }^{12}$ These findings have helped promote the development of smoking cessation programmes directed at pregnant women. ${ }^{13}$ Entry to such specialist cessation services in Scotland, however, usually depends on self reporting of smoking at the maternity booking visit. ${ }^{14}$ Therefore, unless a woman admits to being a current smoker at maternity booking she will not be referred to specialist smoking cessation services and will not receive appropriate support, putting her own health and the health of her unborn child at risk. This study aimed to assess the robustness of self reporting at maternity booking as a method to set targets and to identify pregnant smokers.

Few studies have looked at the variation in accuracy of self reported smoking by deprivation, although the education level of pregnant women has been linked to the accuracy of self reporting. ${ }^{15}$ We hypothesised that self reporting is less effective at identifying smokers in the most deprived areas compared with identifying those in more affluent areas, and that this further compounds existing health disparities during pregnancy. ${ }^{16}$

We compared the routinely collected self reported smoking status of pregnant women with their smoking status validated by means of serum cotinine measurement in order to: use the validated smoking prevalence in the study population to estimate the true smoking prevalence in pregnant women in Scotland; to identify the number of pregnant women in the study population who smoked but had no access to smoking cessation services in order to estimate the number of such women in Scotland; and to identify any variation in the level of under-reporting of smoking by area deprivation and determine what impact this will have on existing health inequalities in pregnancy.

\section{METHODS}

\section{Sample}

The records of all women in the West of Scotland who opted for prenatal screening in the second trimester for Down's syndrome and neural tube defects between May 2003 and July 2004 (that is, likely to result in a 2004 birth) were matched with their obstetric records from the Scottish Morbidity Records (SMR02) (data for 2004, provided by NHS Information Services Division, www.isdscotland.org). The prenatal screening process involves measuring maternal serum $\alpha$ fetoprotein and human chorionic gonadotrophin. Excess serum is stored at $-20^{\circ} \mathrm{C}$. At the time of linking, 2004 was the most recent complete year in the SMR02 dataset. The SMR02 data contain self reported smoking information collected by the midwife at the maternity booking appointment, usually carried out at 8-12 weeks of gestation, for all women delivering in an NHS facility. Women were asked for their smoking status and were recorded as current, former, or never smokers (or unknown if the response was not recorded). Information on the baby's date of birth, mother's date of birth, maternal area deprivation
(Scottish Index of Multiple Deprivation, which is based on postcode of residence reflecting material deprivation) ${ }^{17}$ and date of booking was also available in the SMR02 data. Second trimester prenatal screening was usually carried out at 15-16 weeks of gestation. The records were matched using the mother's surname, forename, date of birth, and hospital number using probability matching techniques. ${ }^{18}$ After data linkage, the database was returned with all patientidentifiable information removed and samples selected for cotinine measurement.

A simple random sample of linked records was selected for cotinine analysis from the births in the 2004 calendar year.

\section{Sample size}

A sample size of 3200 records allowed a 3\% difference in the proportion of cotinine validated smoking and self reported smoking to be detected in the sample as a whole and a difference of $3 \%$ to be detected when comparing the combined deprivation categories 1 and 2 with the combined categories 4 and 5 . We made the a priori decision to collapse the two highest and two lowest deprivation categories to give a greater power with the chosen sample size. To allow for technical difficulties (such as insufficient serum, etc), 3550 women were randomly selected from the linked dataset for cotinine analysis.

\section{Cotinine analysis}

As cotinine is derived only from nicotine metabolism, its measurement in serum is a good indicator of recent nicotine exposure. Cotinine testing was carried out on thawed serum samples at the West of Scotland Regional Genetics Service laboratories using commercially available kits (Cozart STD Micro-Plate Cotinine EIA), and those performing the tests were blind to the women's reported smoking status. Samples were assayed in singletons only. Samples with cotinine concentrations between 10 and $30 \mathrm{ng} / \mathrm{ml}$ (close to the cutoff value) were re-assayed and the mean of the two values taken as the final cotinine concentration. Cotinine concentrations $\geq 13.7 \mathrm{ng} / \mathrm{ml}$ were taken to indicate current smoking. ${ }^{19}$

\section{Statistical analysis}

The prevalences of cotinine validated current smoking and self reported current smoking were determined for the whole sample, and by area deprivation categories, and statistically compared (one-sample test of equality of proportions) using STATA (version 8).

The number of cotinine validated smokers not captured by self reporting (referred to as undetected smokers) was determined by identifying women with never, former, or unknown smoking status who had cotinine values $\geq 13.7 \mathrm{ng} / \mathrm{ml}$, for the whole sample and by deprivation category.

The degree to which the study sample represents the population from which the sample was drawn and the population of pregnant women in Scotland was 
Table 1| Basic characteristics of 3475 pregnant women in the West of Scotland and their subsequent babies. (Values are numbers (percentages) unless stated otherwise)

\begin{tabular}{lc}
$\begin{array}{l}\text { Characteristic } \\
\text { Self reported smoking status in pregnancy: }\end{array}$ \\
\hline Current smoker & $839(24.1)$ \\
\hline Former smoker & $367(10.6)$ \\
\hline Never smoker & $1971(56.7)$ \\
\hline Unknown & $298(8.6)$ \\
\hline Mean (SD) maternal age (years)* & $29.4(6.0)$ \\
\hline Mean (SD) gestation at delivery (weeks) & $39.2(2.0)$ \\
\hline Mean (SD) birth weight of baby (g) & $3390.8(600.0)$ \\
\hline Deprivation categoryt: & $440(12.7)$ \\
\hline 1 (least deprived) & $545(15.7)$ \\
\hline 2 & $733(21.1)$ \\
\hline 3 & $730(21.0)$ \\
\hline 4 & $1023(29.5)$ \\
\hline 5 (most deprived)
\end{tabular}

*Data missing for one record.

†Based on Scottish Index of Multiple Deprivation. Data missing for four records.

determined by comparing the distribution of maternal age, deprivation category, and self reported smoking status in the study sample with the population of pregnant women in the West of Scotland who opted to be screened, the total pregnant population in the West of Scotland (including those who did not opt for screening), and the population of pregnant women in Scotland. Cotinine validated smoking prevalence in the study sample was used to estimate the true smoking prevalence in the population of pregnant women in Scotland, accounting for differences in the distribution of deprivation, maternal age, and self reported smoking status using standardisation techniques. For example, the estimated number of cotinine validated pregnant smokers in Scotland aged $<20$ in deprivation categories 1 and 2 who reported never smoking would be calculated by multiplying the proportion of such women in the study sample by the number of pregnant women aged $<20$ in deprivation categories 1 and 2 reporting never smoking in the Scottish pregnant population. Scottish Morbidity Records (SMR02) data were used to project figures for Scotland.

The number of undetected smokers was estimated by subtracting the number of smokers identified by self reporting from the estimated number of true smokers. Fifty women with no data on deprivation category or age were excluded, leaving 52591 pregnant women in Scotland with a 2004 birth. In generating the projected estimates for Scotland, we collapsed the maternal age categories (to $<24,25-29,30-34$ and $\geq 35$ years) and the highest and lowest deprivation categories $(1+2$ and $4+5)$ because of small numbers in the sample population.

Statistical significance of differences between categorical variables was determined with Pearson's $\chi^{2}$ test. All analyses were performed with SPSS (version 15) or STATA (version 8). To explore the sensitivity of the findings to the cotinine concentration we used in this study to denote a current smoker, we repeated the analyses using alternative cotinine levels found in the literature.

\section{Ethical approval and data protection}

We contacted the Central Office for Research Ethics Committees (corec.org.uk), which advised that ethical approval was not needed for the study. Data protection issues were discussed with the data protection officer at the University of Glasgow and a confidentiality statement for use of NHS patient data was completed. The Privacy Advisory Committee of the Information Services Division (ISD) of NHS National Services approved the study for linkage with routinely collected data held by ISD.

\section{RESULTS}

Of the 29975 women in the West of Scotland who gave birth in 2004, $21029(70 \%)$ opted for second trimester screening. Of these, $97 \%$ could be linked to their obstetric SMR02 data and 3550 were randomly selected for cotinine analysis; $98 \%$ of samples were located and assayed (fig 1). Seventy one serum samples with cotinine concentration of $10-30 \mathrm{ng} / \mathrm{ml}$ (close to the cut-off value) were re-analysed.

Over half of the 3475 women in the sample reported never smoking, and just less than a quarter reported being current smokers. The self reported smoking status was unknown for $9 \%$ of the study population (table 1). The profile of maternal age, baby's birth weight, and gestation at delivery were all typical of that seen in Scotland. ${ }^{20}$ Women in deprived areas were over-represented in this sample, as in the West of Scotland. ${ }^{17}$

\section{Under-reporting of smoking in the sample population} According to the figures for self reported smoking status, $839(24.1 \%)$ of the pregnant women were current smokers. This value is significantly lower than the cotinine validated estimate of $1046(30.1 \%)$ who were current smokers (table 2). As expected, the prevalence of smoking in the most deprived categories was greater than in the least deprived categories, with both the self reported and cotinine validated estimates. However, the difference between the cotinine validated and self reported smoking estimates is greater in the

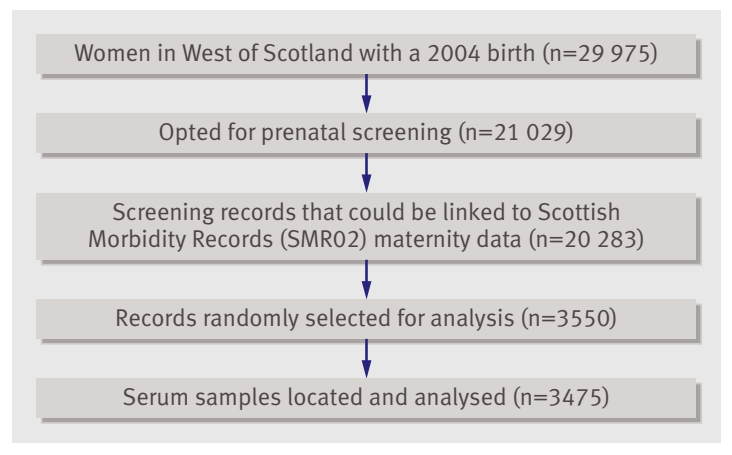

Fig 1| Selection of study sample for cotinine analysis 
Table 2|Prevalence of current smoking among 3475 pregnant women in the West of Scotland according to self reported smoking and cotinine validated smoking

\begin{tabular}{|c|c|c|c|c|c|c|c|}
\hline & \multicolumn{2}{|c|}{ Self reported smoking* } & \multicolumn{2}{|c|}{ Cotinine validated smoking $\dagger$} & \multicolumn{3}{|c|}{ Difference } \\
\hline \multicolumn{8}{|l|}{ Deprivation categorył: } \\
\hline $1+2(n=985)$ & 101 & $10.3(8.5$ to 12.3$)$ & 142 & 14.4 (12.3 to 16.7$)$ & 4.1 & 4.23 & $<0.001$ \\
\hline
\end{tabular}

*Measured at booking appointment (8-12 weeks of gestation).

†Measured at about 15 weeks of gestation. Cotinine concentrations $\geq 13.7 \mathrm{ng} / \mathrm{ml}$ were taken to indicate current smoking.

$\ddagger$ Based on Scottish Index of Multiple Deprivation (categories $1+2$ are least deprived, $4+5$ are most deprived).

most deprived groups than in the least deprived groups (table 2).

Sixty one $(7 \%)$ of the self reported smokers had cotinine concentrations below the cut-off value for current smoking, and were therefore classified as non-smokers (table 3). These women could have quit between their booking and screening appointments, have been light smokers, or be recording errors.

The proportion of cotinine validated current smokers who were correctly identified by self reporting was $74.4 \%$. This means that $25.6 \%(\mathrm{n}=268)$ of cotinine validated smokers were not detected by self reporting and were therefore not offered smoking cessation services (table 4). The number of such undetected smokers was about three times greater in the most deprived areas than in the least deprived areas. However, this represented a smaller proportion of the total number of cotinine validated smokers in the deprived areas than in the affluent areas; it seems that a greater proportion of women in affluent areas failed to report their smoking compared with women in deprived areas.

Although not the focus of this study, the proportion of cotinine validated smokers in the "unknown" category for self reported smoking broadly represented the distribution across the whole study population.

\section{Projected smoking figures for Scotland}

We determined the degree to which our sample was representative of the screened population, the West of Scotland population (from which the sample was drawn), and the Scottish population of pregnant women (table 5). We found no differences between the sample population and the screened population in any of the characteristics examined. Comparing the sampled population with the West of Scotland population, there were no significant differences in the distribution of self reported smoking or maternal age, but there were small but significant differences by area deprivation. The distribution of maternal age within deprivation categories was also compared across populations and no differences were seen between the sampled and West of Scotland populations. Comparing the sampled population with the Scottish population, there was a significant difference in area deprivation and self reported smoking but not maternal age. These data showed the need to adjust at least for differences in self reported smoking and area deprivation when generating projected figures for Scotland. Although no differences in the maternal age distribution were detected, projected figures were also adjusted for maternal age to account for any residual confounding that might arise between age categories.

After adjustment for the distribution of area deprivation, self reported smoking, and maternal age, we estimated the number of pregnant smokers in Scotland to be 14521 (that is, a smoking prevalence of $27.6 \%$ ), and 2400 of these would be undetected by self report, representing $4.6 \%$ of the total population of pregnant women in Scotland. The number of undetected smokers in the most deprived areas (deprivation categories $4+5, \mathrm{n}=1196)$ is nearly twice that in the least deprived areas (categories $1+2, n=642$ ). Using the projected figures for Scotland, the prevalence of smoking in the most deprived areas is $39 \%$ compared with $14 \%$ in the least deprived areas.

The distribution of cotinine values produced two quite distinct populations (see figure in the extra material on bmj.com), suggesting that the findings were robust to the chosen cotinine cut-off value of $13.7 \mathrm{ng} /$ $\mathrm{ml}$. Using an alternative cut-off of $24 \mathrm{ng} / \mathrm{ml}^{21}$ produced a prevalence of cotinine validated smoking for the study population of $29.3 \%$ ( $\mathrm{n}=1018)$, very similar to the $30.1 \%$ produced using the original cut-off (see extra material on bmj.com for all other data using the alterative cut-off value).

\section{DISCUSSION}

Using cotinine validation to identify smokers, we estimate that 1046 of the 3475 women in the study population smoked during pregnancy, which is $25 \%$ higher than figures based on self reported smoking (839/

Table $3 \mid$ Proportion of pregnant women with serum cotinine concentration above and below the cut-off value for current smoking by their self reported smoking status. (Values are numbers (percentages))

\begin{tabular}{lcc} 
& \multicolumn{2}{c}{ Serum cotinine concentration } \\
\cline { 2 - 3 } Self reported smoking status & $\langle 13.7 \mathrm{ng} / \mathrm{ml}$ & $\geq 13.7 \mathrm{ng} / \mathrm{ml}$ \\
Current $(\mathrm{n}=839)$ & $61(7)$ & $778(93)$ \\
\hline Former $(\mathrm{n}=367)$ & $273(74)$ & $94(26)$ \\
\hline Never $(\mathrm{n}=1971)$ & $1875(95)$ & $96(5)$ \\
\hline Unknown $(\mathrm{n}=298)$ & $220(74)$ & $78(26)$
\end{tabular}


Table $4 \mid$ Cotinine validated current smoking among pregnant women that was undetected (not captured by self reported smoking status)

\begin{tabular}{lccc} 
& No of cotinine validated smokers & $\begin{array}{c}\text { No }(\%) \text { of cotinine validated smokers } \\
\text { not captured by self reporting }\end{array}$ & $\begin{array}{c}\text { Undetected smokers as \% } \\
\text { of all pregnant women }\end{array}$ \\
\hline Total sample $(n=3475)$ & 1046 & $268(25.6)$ & 7.7 \\
\hline Deprivation category*: & 142 & $56(39.4)$ & $5.7 \dagger$ \\
\hline $1+2(n=985)$ & 706 & $155(22.0)$ & 8.8
\end{tabular}

*Based on Scottish Index of Multiple Deprivation (categories 1+2 are least deprived, 4+5 are most deprived).

$\dagger z=0.293, P=0.003$ for difference in distribution of undetected smokers across deprivation categories $1+2$ and $4+5$.

3475). The projected true smoking prevalence for pregnant women in Scotland (after adjusting for area deprivation, maternal age, and self reported smoking) is $28 \%$, notably higher than the $23 \%$ based on self reporting. ${ }^{2}$ Projected figures suggest that in Scotland each year more than $17 \%$ of pregnant smokers $(\mathrm{n}=2400)$ are not identified as such and are therefore not offered smoking cessation services.

There was a striking difference in smoking prevalence in pregnant women between area deprivation categories, reflecting that seen elsewhere. ${ }^{22}$ Nearly $40 \%$ of smokers in the least deprived areas (deprivation categories $1+2$ ) did not report their smoking status compared with only $22 \%$ of smokers in the most deprived areas (categories 4+5). This possibly reflects a greater expectation in more affluent areas that women will quit smoking during pregnancy. However, because of the larger number of smokers in deprived areas, in absolute terms, there were three times as many undetected smokers in the most deprived areas compared with the least deprived areas in the sample population.

In our sample, about a quarter of validated smokers went undetected. This proportion is similar to that seen in other studies of pregnant women in the $\mathrm{UK}^{19}$ and elsewhere ${ }^{6-823}$ Higher proportions of undetected smokers have been seen: in one US study more than $50 \%$ of cotinine validated smokers were undetected by self reporting. ${ }^{24}$ High proportions of undetected smokers (the proportion of smokers who do not report their smoking) are commonly seen in pregnant populations involved in cessation programmes, ${ }^{2526}$ as might be expected. Other studies have reported lower proportions of undetected smokers; ${ }^{22} 27$ some as low as $1 \%{ }^{28}$ The variation in proportion of undetected smokers can be largely explained by different populations and settings in which smoking status was reported. The study that reported only $1 \%$ undetected smokers was based on a Swedish population in which only $8 \%$ of the pregnant population were smokers, ${ }^{28}$ whereas the study reporting over 50\% undetected smokers examined a population in a deprived area with a smoking prevalence of $35 \%$.

There is general agreement that serum cotinine is ideal for measuring smoking status because of its long half life ( 9 hours in pregnant women ${ }^{29}$ and the procedure's optimised sensitivity $(94 \%)$ and specificity $(81 \%) .{ }^{419}$ There is little variation in the cotinine cutoff level used to define a pregnant smoker, with some studies using the lower value of $10 \mathrm{ng} / \mathrm{ml}^{727}$ and others using values up to $24 \mathrm{ng} / \mathrm{ml} .^{821}$ The results of our study were robust to the chosen cut-off value. Cotinine is specific for nicotine, but not necessarily for smoking; both nicotine replacement therapy and exposure to environmental tobacco smoke have been shown to elevate serum cotinine levels. The median cotinine level recorded in studies measuring the impact of exposure to environmental tobacco smoke is well below our chosen cut-off value of $13.7 \mathrm{ng} / \mathrm{ml}$ - a median cotinine concentration of $4 \mathrm{ng} / \mathrm{ml}$ has been reported for office staff $^{30}$ and $8 \mathrm{ng} / \mathrm{ml}$ for bar staff. ${ }^{31}$ Therefore, women exposed to environmental tobacco smoke were unlikely to be miscoded as smokers in our study. The women in this study were pregnant in 2003-4, and nicotine replacement therapy was not routinely recommended in the West of Scotland until two specialist smoking cessation programmes began offering it in $2002(\mathrm{CATCH})^{32}$ and 2004 (BREATHE). ${ }^{33}$ The only service offering nicotine replacement therapy during the time the study women were pregnant was $\mathrm{CATCH}$, which offered it to 65 women. Even if all these 65 women used nicotine replacement products the small number of women involved would be unlikely to bias our findings greatly. In addition, a smoking cessation study based in Glasgow during 2001-3 showed that only $0.8 \%(6 / 718)$ of women reported taking nicotine replacement therapy, ${ }^{34}$ again suggesting that any effects of nicotine replacement therapy in the study population would be minimal.

The recording of self reported smoking status at the antenatal clinic booking visit usually took place about three weeks before the collection of blood used for cotinine analysis. It was possible, therefore, for women to have quit smoking and correctly report being a former smoker at the booking appointment but to resume smoking before the blood was collected. Therefore, it should be acknowledged that some of the unreported smokers may have been former smokers who relapsed after reporting their smoking status and not smokers deliberately denying their smoking status. This distinction may not be so important for the objective of this study-determining the true prevalence of smoking among pregnant women. It may be that, within reason, the later in pregnancy that smoking is measured the more accurate it will be in terms of recording the true number of pregnant smokers - in that it will capture more of those that relapse. ${ }^{23}$ As a result, these data will slightly overestimate the number of current 
Table 5 Distribution of maternal age, area deprivation, and self reported smoking status among pregnant women in the study sample, the screened sample, the West of Scotland, and Scotland. (Values are numbers (percentages) unless stated otherwise)

\begin{tabular}{|c|c|c|c|c|}
\hline \multirow[b]{2}{*}{ All } & \multicolumn{4}{|c|}{ Pregnant women (2004 birth)* } \\
\hline & $\begin{array}{l}\text { Study sample } \\
\quad(n=3475)\end{array}$ & $\begin{array}{c}\text { Screened } \\
\text { population } \\
(n=20283 \dagger)\end{array}$ & $\begin{array}{l}\text { West of Scotland } \ddagger \\
\quad(n=29975)\end{array}$ & $\begin{array}{l}\text { Scotland } \\
(n=52862)\end{array}$ \\
\hline \multicolumn{5}{|l|}{ Self reported smoking status§ } \\
\hline Current & $839(24)$ & $4896(24)$ & $7303(25)$ & $12366(24)$ \\
\hline Former & $367(11)$ & $2072(10)$ & $2770(9)$ & $4712(9)$ \\
\hline Never & $1971(57)$ & $11353(56)$ & $16980(57)$ & $30680(59)$ \\
\hline Unknown & $298(9)$ & $1962(10)$ & $2614(9)$ & $4074(8)$ \\
\hline Difference from study sample & - & $X^{2}=4.4, P=0.2$ & $X^{2}=5.5, P=0.1$ & $X^{2}=13.3, P=0.004$ \\
\hline \multicolumn{5}{|c|}{ Deprivation category (Scottish Index of Multiple Deprivation) } \\
\hline $1+2$ & $985(28)$ & $5683(28)$ & $8738(29)$ & $19621(37)$ \\
\hline 3 & $733(21)$ & $4028(20)$ & $5601(19)$ & 9825 (19) \\
\hline $4+5$ & $1753(51)$ & $10531(52)$ & $15636(52)$ & $23145(44)$ \\
\hline Difference from study sample & - & $X^{2}=4.5, P=0.3$ & $x^{2}=17.4, P<0.002$ & $x^{2}=130, P<0.001$ \\
\hline \multicolumn{5}{|c|}{ Maternal age (years) by deprivation category } \\
\hline \multicolumn{5}{|l|}{ Categories 1+2: } \\
\hline$<20$ & $21(2)$ & $140(3)$ & $235(3)$ & $528(3)$ \\
\hline $20-24$ & $74(8)$ & $505(9)$ & $770(9)$ & $1753(9)$ \\
\hline $25-29$ & $220(22)$ & $1243(22)$ & $1836(21)$ & $4360(22)$ \\
\hline 30-34 & $390(40)$ & $2246(40)$ & $3455(40)$ & $7744(40)$ \\
\hline $35-39$ & $244(25)$ & $1354(24)$ & $2085(24)$ & $4395(22)$ \\
\hline$\geq 40$ & $36(4)$ & $193(3)$ & $357(4)$ & $764(4)$ \\
\hline Difference from study sample & - & $X^{2}=2.8, P=0.7$ & $X^{2}=4.2, P=0.5$ & $X^{2}=5.7, P=0.3$ \\
\hline \multicolumn{5}{|l|}{ Category 3: } \\
\hline$<20$ & $38(5)$ & $188(5)$ & $303(5)$ & $586(6)$ \\
\hline 20-24 & $117(16)$ & $642(16)$ & $882(16)$ & $1629(17)$ \\
\hline $25-29$ & $188(26)$ & $1084(27)$ & $1452(26)$ & $2568(27)$ \\
\hline $30-34$ & $230(31)$ & $1270(32)$ & $1835(33)$ & $3068(32)$ \\
\hline $35-39$ & $138(19)$ & $741(18)$ & $959(17)$ & $1529(16)$ \\
\hline$\geq 40$ & $22(3)$ & $101(3)$ & $170(3)$ & $306(3)$ \\
\hline Difference from study sample & - & $X^{2}=1.4, P=0.9$ & $X^{2}=1.6, P=0.9$ & $X^{2}=5.4, P=0.4$ \\
\hline \multicolumn{5}{|l|}{ Categories 4+5: } \\
\hline$<20$ & $215(12)$ & 1205 (12) & 1907 (12) & $3026(13)$ \\
\hline $20-24$ & $430(25)$ & $2672(25)$ & $4052(26)$ & $6318(27)$ \\
\hline $25-29$ & $464(27)$ & $2774(26)$ & $4018(26)$ & $5922(25)$ \\
\hline $30-34$ & $411(24)$ & $2434(23)$ & $3575(23)$ & $5211(22)$ \\
\hline $35-39$ & $197(11)$ & $1258(12)$ & $1763(11)$ & $2444(10)$ \\
\hline$\geq 40$ & $35(2)$ & $177(2)$ & $321(2)$ & $450(2)$ \\
\hline Difference from study sample & - & $X 2=2.9, P=0.7$ & $X^{2}=1.8, P=0.9$ & $X^{2}=7.2, P=0.2$ \\
\hline \multicolumn{5}{|l|}{ All categories combined: } \\
\hline Difference from study sample & - & $X^{2}=3.1, P=0.7$ & $X^{2}=4.4, P=0.5$ & $X^{2}=3.6, P=0.6$ \\
\hline
\end{tabular}

*Excluding home births and births at non-NHS hospitals.

tRefers to those screening records that could be linked with their obstetric records from the Scottish Morbidity Records (SMR02).

$\ddagger$ Made up of Argyll and Clyde, Ayrshire and Arran, Dumfries and Galloway, Forth Valley, Greater Glasgow, Highland, Lanarkshire, and Western Isles health boards.

§Smoking figures for West of Scotland are for 2005.

ПCollapsed categories are used for $\mathrm{X}^{2}$ test.

Missing data: Sample population-4 records with no deprivation data, 1 record with no maternal age; screened population-18 records with no maternal age, 787 (3.7\%) with no deprivation data, 746 with no self reported smoking data (that is, not linked records); West of Scotland-38 with no region data; Scottish data-163 with no deprivation data.
Extrapolation of the sample results to all pregnant smokers in Scotland is based on two assumptions, the first being that the study population accurately represents the population from which it was drawn (West of Scotland). The study sample is similar to the screened sample in all characteristics tested, which suggests successful random selection of the sample from the screened population. Differences between the study population and the West of Scotland will therefore reflect differences between the screened and nonscreened women. Only if such differences are also related to the accuracy of self reported smoking will there be potential to introduce bias; differences that differentiate screened and non-screened women that relate only to self reported smoking status but not the accuracy of self reporting will not introduce bias. The data suggest that neither self reported smoking status nor maternal age were related to the decision to opt for screening in this setting (table 5), as the study sample and the population of the West of Scotland have similar profiles. A major strength of these data is the high screening rate $(70 \%$ of all women were screened), reducing the potential for differences between the screened and target population. Indeed the lack of a maternal age effect is consistent with the continued monitoring of this screened population. The sample population had a slightly different area deprivation profile compared with the population of the West of Scotland (52\% in the West of Scotland from deprivation categories $4+5$ compared with $51 \%$ of the sample population). However, there is little difference in the percentages; the significance of the result is related to the large sample size rather than an important difference in deprivation profile. Other factors that may be related to a decision to opt for screening include ethnicity and religion. Ethnicity is unlikely to have a large impact on the data as there was very little representation of black and ethnic minority populations in Scotland at the time of data collection ( $2 \%$ non-white people in the 2001 census, General Register Office for Scotland). Catholic women are likely to be underrepresented in the screened population. Although there are substantial numbers of Catholics in Scotland (about $16 \%$ at the 2001 census), it is unlikely that the accuracy of self reported smoking status provided by pregnant Catholic women would be substantially different from that of the rest of the population and therefore these factors are unlikely to introduce bias. There may be yet other differences between the population of screened and non-screened women that we were not able to investigate that also relate to the accuracy of self reported smoking. However, given the high screening rate $(70 \%)$, these differences are unlikely to bias the findings significantly.

The second assumption made is that differences between the West of Scotland and the Scottish population that also relate to the accuracy of self report are accounted for in generating the projected figures. Differences between the pregnant women from these two populations are expected because the West of Scotland includes some of the more deprived areas of the smokers who were not offered smoking cessation services because, at the time of asking, the to-be relapsers were in fact former smokers, although they are arguably in need of such services. 


\section{WHAT IS ALREADY KNOWN ON THIS TOPIC}

Self reported smoking during pregnancy is known to be an inaccurate method of identifying smokers

Self reported smoking in Scotland is used to generate smoking prevalence and, largely, to target smoking cessation services

\section{WHAT THIS STUDY ADDS}

Reliance on self reported smoking during pregnancy underestimates the true smoking prevalence in Scotland by $17 \%$

Each year in Scotland twice as many pregnant smokers from more deprived areas go undetected compared with pregnant smokers in the least deprived areas

Reliance on self reporting results in a failure to detect over 2400 pregnant smokers each year in Scotland who are therefore not offered smoking cessation services

country. The projected figures take account of area deprivation, smoking status, and maternal age, which probably accounts for the major differences between east and west Scotland. Again religion (Catholicism in the West of Scotland) is unlikely to be related to the accuracy of self report.

Some errors in the recording or transcribing of the self reported smoking status at the booking appointment will have happened. It is not likely that these recording errors would be systematic (that is, by deprivation or smoking status), and such errors are therefore unlikely to bias these finding away from the null hypothesis. The recording of self reported smokers with a serum cotinine concentration below the cut-off value for non-smokers may slightly underestimate the true prevalence of smokers.

For $9 \%$ of the routinely collected data used for this study there was no valid information about self reported smoking status (the information was either not requested or not recorded), compounding the problem of inaccurate self reported data. There is some evidence that the issue of smoking is not necessarily given high priority in the relationship between midwife and expectant mother, ${ }^{14}$ possibly explaining cases of missing data. Similar problems with data quality have also been highlighted with routinely collected self reported data in other regions. ${ }^{35}$ The poor quality and accuracy of routinely collected self reported smoking data in pregnant women shown in this study and elsewhere ${ }^{35}$ call for better methods of routinely identifying smokers during pregnancy. The current policy in Glasgow is for all women to provide self reported smoking status and undergo a carbon monoxide breath test at maternity booking. If implemented fully, this could increase the identification of pregnant smokers from $75 \%$ to about $95 \% .{ }^{33}$ Further studies are required to determine if offering routine biochemical validation (such as carbon monoxide breath tests) to all pregnant women at maternity booking would be the most cost effective method to identify smokers, to monitor targets, and to increase the reach of specialist smoking cessation support. Accurate smoking information also refines the estimation of individual fetal risks of Down's syndrome by prenatal screening, since maternal smoking causes changes in the levels of the biochemical markers used in the screening test $^{37}$ (discussed further in a subsequent paper).

In conclusion, reliance on self reporting to measure smoking during pregnancy significantly underestimates the number of pregnant smokers in Scotland, with more than 2400 unrecognised pregnant smokers a year who will not be offered smoking cessation services. Reliance on self reporting resulted in twice as many undetected smokers in the most deprived areas compared with the least deprived areas. Overall, these figures call for more accurate methods of identifying pregnant smokers, especially when such data are used to inform policy and provide patient care.

Contributors: DS analysed the data, wrote the manuscript, and contributed to seeking funding. TV performed the cotinine analysis, analysed data, and contributed to drafts of the manuscript. DMT, JAC, and DAA conceived the idea, designed the study, and contributed to the analysis, writing the manuscript, and seeking funding. JC contributed to the analysis, writing the manuscript, and seeking funding. DMT is guarantor for the study.

Funding: This was provided by a Glasgow Centre for Population Health Grant. All authors are independent of the funding body.

Competing interests: None declared.

Ethical approval: Not required.

Data sharing: No additional data available.

1 Giovino GA. The tobacco epidemic in the United States. Am J Prev Med 2007:33:S318-26.

2 Information Services Division, NHS Scotland. Births and babies: smoking and pregnancy. 2009. www.isdscotland.org/isd/2911. html.

3 Scientific Advisory Committee on Nutrition. Infant feeding survey 2005: a commentary on infant feeding practices in the UK. Stationery Office, 2009.

4 Corbet J, MacLeod P, Martin C, Hope S. Scotland's people: results from the 2005 Scottish Household Survey. Annual report. Scottish Executive, 2006.

5 Russell T, Crawford M, Woodby L. Measurements for active cigarette smoke exposure in prevalence and cessation studies: why simply asking pregnant women isn't enough. Nicotine Tob Res 2004;6(suppl 2):S141-51.

6 Ford RP, Tappin DM, Schluter PJ, Wild CJ. Smoking during pregnancy: how reliable are maternal self reports in New Zealand? J Epidemiol Community Health 1997;51:246-51.

7 Klebanoff MA, Levine RJ, Morris CD, Hauth JC, Sibai BM, Ben Curet L, et al. Accuracy of self-reported cigarette smoking among pregnant women in the 1990s. Paediatr Perinat Epidemiol 2001;15:140-3.

8 Lindqvist R, Lendahls L, Tollbom O, Aberg H, Hakansson A. Smoking during pregnancy: comparison of self-reports and cotinine levels in 496 women. Acta Obstet Gynecol Scand 2002;81:240-4.

9 Patrick DL, Cheadle A, Thompson DC, Diehr P, Koepsell T, Kinne S. The validity of self-reported smoking: a review and meta-analysis. Am J Public Health 1994;84:1086-93.

10 Scottish Government. NHS national targets: related to NHS nationa priorities. 2003. www.scotland.gov.uk/Publications/2003/10/ $18432 / 28416$.

11 Department of Health. Tackling health inequalities: targeting routine and manual smokers in support of the Public Service Agreement smoking prevalence and health inequality targets. DH, 2009

12 Lumley J, Oliver SS, Chamberlain C, Oakley L. Interventions for promoting smoking cessation during pregnancy. Cochrane Database Syst Rev 2004;(4):CD001055.

13 Department of Health. Smoking kills: a white paper on tobacco. Cm 4177. DoH, 1998.

14 NHS Health Scotland. Smoking cessation support in pregnancy in Scotland. 2008. www.healthscotland.com/documents/2665.aspx.

15 Parna K, Rahu M, Youngman LD, Rahu K, Nygard-Kibur M, Koupil I. Self-reported and serum cotinine-validated smoking in pregnant women in Estonia. Matern Child Health / 2005;9:385-92.

16 D'Souza L, Garcia I. Improving services for disadvantaged childbearing women. Child Care Health Dev 2004;30:599-611.

17 Carstairs V, Morris R. Deprivation and health in Scotland. Health Bull (Edinb) 1990;48:162-75.

18 Kendrick S, Clarke J. The Scottish record linkage system. Health Bull (Edinb) 1993;51:72-9. 
19 Jarvis MJ, Tunstall-Pedoe H, Feyerabend C, Vesey C, Saloojee Y. Comparison of tests used to distinguish smokers from nonsmokers. Am J Public Health 1987;77:1435-8.

20 Information Services Division, NHS Scotland. Births and babies: birthweight \& gestation, singletons. 2009. www.isdscotland.org/ isd/1461.html.

21 Boyd NR, Windsor RA, Perkins LL, Lowe JB. Quality of measurement of smoking status by self-report and saliva cotinine among pregnant women. Matern Child Health J 1998;2:77-83.

22 Owen L, McNeill A. Saliva cotinine as indicator of cigarette smoking in pregnant women. Addiction 2001;96:1001-6.

23 England LJ, Grauman A, Qian C, Wilkins DG, Schisterman EF, Yu KF, et al. Misclassification of maternal smoking status and its effects on an epidemiologic study of pregnancy outcomes. Nicotine Tob Res 2007;9:1005-13.

24 Webb DA, Boyd NR, Messina D, Windsor RA. The discrepancy between self-reported smoking status and urine cotinine levels among women enrolled in prenatal care at four publicly funded clinical sites. J Public Health Manag Pract 2003;9:322-5.

25 Kendrick JS, Zahniser SC, Miller N, Salas N, Stine J, Gargiullo PM, et al. Integrating smoking cessation into routine public prenatal care: the Smoking Cessation in Pregnancy project. Am J Public Health 1995;85:217-22.

26 Windsor RA, Lowe JB, Perkins LL, Smith-Yoder D, Artz L, Crawford M, et al. Health education for pregnant smokers: its behavioral impact and cost benefit. Am J Public Health 1993;83:201-6.

27 McDonald SD, Perkins SL, Walker MC. Correlation between self reported smoking status and serum cotinine during pregnancy. Addict Behav 2005;30:853-7.
28 George L, Granath F, Johansson AL, Cnattingius S. Self-reported nicotine exposure and plasma levels of cotinine in early and late pregnancy. Acta Obstet Gynecol Scand 2006;85:1331-7.

29 Dempsey D, Jacob P III, Benowitz NL. Accelerated metabolism of nicotine and cotinine in pregnant smokers. J Pharmacol Exp Ther 2002;301:594-8.

30 Hammond SK, Sorensen G, Youngstrom R, Ockene JK. Occupational exposure to environmental tobacco smoke. JAMA 1995;274:956-60.

31 Jarvis MJ, Foulds J, Feyerabend C. Exposure to passive smoking among bar staff. Br J Addict 1992;87:111-3.

32 Bryce A, Butler C, Gnich W, Sheehy C, Tappin DM. CATCH: development of a home-based midwifery intervention to support young pregnant smokers to quit. Midwifery 2009;25:473-82.

33 McGowan A, Hamilton S, Barnett D, Nsofor M, Proudfoot J, Tappin DM. 'Breathe': the stop smoking service for pregnant women in Glasgow. Midwifery 2008 Aug 7 [epub ahead of print].

34 Tappin DM, Lumsden MA, Gilmour WH, Crawford F, McIntyre D, Stone $\mathrm{DH}$, et al. Randomised controlled trial of home based motivational interviewing by midwives to help pregnant smokers quit or cut down. BMJ 2005;331:373-7.

35 O'Gorman C. We need better data on smoking in pregnancy. BMJ 2008;336:330.

36 Usmani ZC, Craig P, Shipton D, Tappin D. Comparison of CO breath testing and women's self-reporting of smoking behaviour for identifying smoking during pregnancy. Substance Abuse Treat, Prev Policy 2008;3:4

37 Aitken DA, Crossley JA, Spencer K. Prenatal screening for neural tube defects and aneuploidy. In: Rimoin DL, Connor JM, Pyeritz RE, Korf BR, eds. Principles and practice of medical genetics. Churchill Livingstone, 2007:636-78.

Accepted: 6 July 2009 